\title{
ON THE BALAYAGE FOR LOGARITHMIC POTENTIALS
}

\author{
NOBUYUKI NINOMIYA
}

To Professor KIyoshi Noshiro on the occasion of his 60 th birthday

In this paper, we shall consider the logarithmic potential

$$
U^{\mu}(P)=\int \log \frac{1}{P Q} d \mu(Q),
$$

where $\mu$ is a positive measure in the plane, $P$ and $Q$ are any points and $P Q$ denotes the distance from $P$ to $Q$. In general, consider the potential

$$
K(P, \mu)=\int K(P, Q) d \mu(Q)
$$

of a positive measure $\mu$ taken with respect to a kernel $K(P, Q)$ which is a continuous function in $P$ and $Q$ and may be $+\infty$ for $P=Q$. A kernel $K(P, Q)$ is said to satisfy the balayage principle if, given any compact set $F$ and any positive measure $\mu$ with compact support, there exists a positive measure $\mu^{\prime}$ supported by $F$ such that $K\left(P, \mu^{\prime}\right)=K(P, \mu)$ on $F$ with a possible exception of a set of $K$-capacity zero and $K\left(P, \mu^{\prime}\right) \leqq K(P, \mu)$ everywhere. A kernel $K(P, Q)$ is said to satisfy the equilibrium principle if, given any compact set $F$, there exists a positive measure $\lambda$ supported by $F$ such that $K(P, \lambda)=V$ (a constant) on $F$ with a possible exception of a set of $K$-capacity zero and $K(p, \lambda) \leqq V$ everywhere. The logarithmic kernel

$$
K(P, Q)=\log \frac{1}{P Q}
$$

satisfies the equilibrium principle in the plane, but it does not satisfy the balayage principle in the above form. As is well-known, given any compact set $F$ and any point $M$ of the complement $C F$ of $F$, there exist a positive measure $\varepsilon^{\prime}$ supported by $F$ with total mass 1 and a non-negative constant $r$ such that (1) $U^{\varepsilon^{\prime}}(P)=\log \frac{1}{M P}+\gamma$ on $F$ with a possible exception of a set of logarithmic capacity zero, and

Received July 1, 1966. 
(2) $U^{\varepsilon^{\prime}}(P) \leqq \log \frac{1}{M P}+\gamma$ everywhere.

Here, the constant $r$ does not always reduce to zero. The balayage for logarithmic potentials has been studied in detail in the book of $\mathrm{C}$. de la Vallee Poussin ([2]). In the present paper, we shall study it in a more general case. Namely we shall try to balayage any positive measure onto any closed set.

We shall deal with the positive measures whose logarithmic potentials are never $-\infty$. The total mass of such a positive measure is naturally finite. The logarithmic potential of such a positive measure is superharmonic in the plane and is harmonic outside the support of the measure. Let us recall the definition of the logarithmic capacity $C(F)$ of a compact set $F$. Putting

$$
V=\inf _{\mu} \sup _{P} U^{\mu}(P) \text { and } W=\inf _{\mu} \int U^{\mu} d \mu
$$

for any positive measure $\mu$ supported by $F$ with total mass 1 , we have always $V=W$. The logarithmic capacity is given by $C(F)=e^{-V}=e^{-W}$ if $V=W<+\infty$ and by $C(F)=0$ if $V=W=+\infty$.

We have the following theorem.

ThEOREM 1. Given any closed set $F$ containing a compact set of positive logarithmic capacity and any positive measure $\mu$ with total mass 1 , there exist a positive measure $\mu^{\prime}$ supported by $F$ with total mass 1 and a non-negative constant $r_{\mu}$ such that

(1) $U^{\mu^{\prime}}(P)=U^{\mu}(P)+\gamma_{\mu}$ on $F$ with a possible exception of a set of logarithmic capacity zero, and

(2) $U^{\mu^{\prime}}(P) \leqq U^{\mu}(P)+\gamma_{\mu}$ everywhere.

We shall call $\mu^{\prime}$ a balayaged measure of $\mu$ onto $F$. We can construct a balayaged measure such that the reciprocal relation always holds:

(3) $\int\left(U^{\mu^{\prime}}-\gamma_{\mu}\right) d \nu=\int\left(U^{\nu^{\prime}}-\gamma_{\nu}\right) d \mu$ for any positive measure $\mu$ with total mass 1 and any positive measure $\nu$ of finite logarithmic energy with total mass 1 , where $\mu^{\prime}$ and $\nu^{\prime}$ are their balayaged measures and $\gamma_{\mu}$ and $\gamma_{\nu}$ are their associated constants.

Under this additional condition, a balayaged measure is unique.

Proof. We are going to prove the theorem by dividing the proof into several steps.

[I] The case where $F$ is compact and the support of $\mu$ is a compact set which has no intersection with $F$. 
Let us consider the Gauss variation

$$
G(\nu)=\iint \log \frac{1}{P Q} d_{\nu}(Q) d_{\nu}(P)-2 \int U^{\mu}(P) d_{\nu}(P)
$$

for any positive measure $\nu$ supported by $F$. Put

$$
G^{*}=\inf _{\nu} G(\nu)
$$

for the positive measures $\nu$ supported by $F$ with total mass 1 . There exists a sequence of positive measures $\nu_{n}$ supported with total mass 1 such that $G\left(\nu_{n}\right) \downarrow G^{*}$. We may suppose that $\left\{\nu_{n}\right\}$ je a va uely convergent sequence by selecting a partial sequence in advance if necesiry. The limiting measure $\mu^{\prime}$ is a positive measure supported by $F$ with total mass 1 . As $U^{\mu}(P)$ is a finite and continuous function on $F$, we have

$$
G^{*} \leqq G\left(\mu^{\prime}\right) \leqq \lim _{n \rightarrow+\infty}\left(\cdots=G^{*} .\right.
$$

So, we have $G^{*}=G\left(\mu^{\prime}\right)$. As is well-knowi: $\left.[1], \S 37\right)$, in putting

$$
r=\int_{F}\left(U^{\mu^{\prime}}-U^{\mu}\right) d \mu^{\prime}
$$

we have

(1) $U^{\mu^{\prime}}(P) \geqq U^{\mu}(P)+\gamma$ on $F$ with a possible exception of a set of logarithmic capacity zero, and

(2) $U^{\mu^{\prime}}(P) \leqq U^{\mu}(P)+\gamma$ on the support of $\mu^{\prime}$.

Let us show that the latter inequality holds everywhere. In fact, the function

$$
f(P)=U^{\mu^{\prime}}(P)-U^{\mu}(P)-\gamma
$$

is subharmonic in each component of the complement $C F^{\prime}$ of the support $F^{\prime}$ of $\mu^{\prime}$, and we have

$$
\lim _{P \rightarrow M} U^{\mu^{\prime}}(P) \leqq \lim _{Q \rightarrow M} U^{\mu^{\prime}}(Q)
$$

at each boundary point $M$ of $F^{\prime}, P$ being points of $C F^{\prime}$ and $Q$ being points of $F^{\prime}$. This is owing to the fact that the logarithmic kernel satisfies the maximum principle: the inequality $U^{\lambda}(P) \leqq K$ (a constant) on the support of a positive measure $\lambda$ induces the same inequality everywhere. $U^{\mu}(P)$ being finite and continuous in a neighbourhood of $F^{\prime}$, we have

$$
\lim _{P \rightarrow M} f(P) \leqq \lim _{Q \rightarrow M} f(Q) \leqq 0
$$


at each boundary point $M$ of $F^{\prime}$. Furthermore, let us notice that $r \geqq 0$. It is because we have

$$
\gamma \geqq \int\left(U^{\mu^{\prime}}-U^{\mu}\right) d \lambda=\int U^{\lambda} d \mu^{\prime}-\int U^{\lambda} d \mu \geqq 0
$$

for the equilibrium measure $\lambda$ with total mass 1 on $F^{\prime}$. So, we have

$$
\varlimsup_{P \rightarrow \infty} f(P)=-r \leqq 0
$$

which is due to $\int d \mu^{\prime}=\int d \mu$. Therefore, we have $f(P) \leqq 0$ in each component of $C F^{\prime}$. Hence, we have

(1) $U^{\mu^{\prime}}(P)=U^{\mu}(P)+r$ on $F$ with a possible exception of a set of logarithmic capacity zero, and

(2) $U^{\mu^{\prime}}(P) \leqq U^{\mu}(P)+r$ everywhere.

It is sufficient to put $\gamma_{\mu}=r$. Let us remark that this balayaged measure $\mu^{\prime}$ is of finite logarithmic energy.

[II] The case where $F$ is compact and $\mu(F)=0$.

$\mu$ is supported by the complement $C F$ of $F$. Let $D_{0}$ be a large disk containing $F$, and $\left\{D_{n}\right\}$ and $\left\{D_{-n}\right\}$ be two sequences of bounded open sets such that

$$
D_{0} \supset D_{-1} \supset D_{-2} \supset \cdots \supset D_{-n} \supset \cdots \rightarrow F
$$

and

$$
D_{0} \subset D_{1} \subset D_{2} \subset \cdots \subset D_{n} \subset \cdots \rightarrow \text { the whole plane. }
$$

Let $\mu_{n}$ be the restricted measure of $\mu$ to

$$
E_{n}=D_{n}-D_{n-1} \quad(n= \pm 1, \pm 2, \pm 3, \ldots) .
$$

The support of $\mu_{n}$ is a compact set which has no intersection with $F$. Let $a_{n}$ be the total mass of $\mu_{n}$ and $\mu_{n}^{\prime}$ be a balayaged measure, with total mass $a_{n}$, of $\mu_{n}$ onto $F$. We have with a non-negative constant $\gamma_{\mu_{n}}$

(1) $U^{\mu_{n}^{\prime}}(P)=U^{\mu_{n}}(P)+\gamma_{\mu_{n}}$ on $F$ with a possible exception of a set of logarithmic capacity zero, and

(2) $U^{\mu_{n}^{\prime}}(P) \leqq U^{\mu_{n}}(P)+\gamma_{\mu_{n}}$ everywhere.

As we have $\mu=\sum \mu_{n}$ and the measure $\mu^{\prime}=\sum \mu_{n}^{\prime}$ is a positive measure supported by $F$ with total mass 1 , the series 


$$
\sum_{n=-\infty}^{+\infty} \gamma_{\mu_{n}}
$$

is convergent. Denoting by $\gamma_{\mu}(\geqq 0)$ the sum of that series, we have

(1) $U^{\mu^{\prime}}(P)=U^{\mu}(P)+\gamma_{\mu}$ on $F$ with a possible exception of a set of logarithmic capacity zero, and

(2) $U^{\mu^{\prime}}(P) \leqq U^{\mu}(P)+\gamma_{\mu}$ everywhere.

Let us remark that this balayaged measure $\mu^{\prime}$ is the sum of positive measures of finite logarithmic energy.

[III] The case where $F$ is compact and $\mu(C F)=0$.

The support of $\mu$ is a compact subset of $F$. Taking a larger number $R$ than the diameter of $F$, put

$$
U_{R}^{\mu}(P)=\int\left(\log \frac{1}{P Q}-\log \frac{1}{R}\right) d \mu(Q) .
$$

We have

$$
\log \frac{1}{P Q}-\log \frac{1}{R}>0 \text { and } U_{R}^{\mu}(P)>0
$$

for any points $P$ and $Q$ of $F$. Let

$$
G_{n}=\left\{P ; U_{R}^{\mu}(P)>n\right\} \text { and } F_{n}=F-G_{n},
$$

and $\mu_{1}$ and $\mu_{2 n}$ be the restricted measures of $\mu$ to $F_{n}$ and $G_{n}$ respectively. As we have

$$
U_{R}^{\mu_{1} n}(P) \leqq n \text { and } U_{R}^{\mu_{2} n}(P) \leqq n
$$

on $F_{n}$, we have

$$
\int U^{\mu_{1} n} d \mu_{1 n}-\log \frac{1}{R}\left(\int d \mu_{1 n}\right)^{2} \leqq n \cdot \int d \mu_{1 n}
$$

and

$$
U^{\mu_{2} n}(P)-\log \frac{1}{R}\left(\int d \mu_{2 n}\right) \leqq n \text { on } F_{n}
$$

So, $\mu_{1 n}$ is of finite logarithmic energy and the logarithmic potential of $\mu_{2 n}$ is bounded on $F_{n}$. Let $a_{n}$ be the total mass of $\mu_{2 n}$ and $\mu_{2}^{\prime}$ be a balayaged measure, with total mass $a_{n}$, of $\mu_{2}$ onto $F_{n}$. We have with a non-negative constant $\gamma_{\mu_{n}}$

(1) $U^{\mu_{2 n}^{\prime}}(P)=U^{\mu_{2 n}}(P)+\gamma_{\mu_{n}}$ on $F_{n}$ with a possible exception of a set of loga- 
rithmic capacity zero, and

(2) $U^{\mu_{n}^{\prime}}(P)=U^{\mu_{2 n}}(P)+r_{\mu_{n}}$ everywhere.

The measure

$$
\mu_{n}^{\prime}=\mu_{1 n}+\mu_{2 n}^{\prime}
$$

is a positive measure supported by $F_{n}$ with total mass 1 and is of finite logarithmic energy. We have

(1) $U^{\mu_{n}^{\prime}}(P)=U^{\mu}(P)+\gamma_{\mu_{n}}$ on $F_{n}$ with a possible exception of a set of logarithmic capacity zero, and

(2) $U^{\mu_{n}^{\prime}}(P) \leqq U^{\mu}(P)+\gamma_{\mu_{n}}$ everywhere.

Let us prove that $U^{\mu_{n}^{\prime}}(P)-\gamma_{\mu_{n}}$ increases with $n$ everywhere. Let $P$ be any point of $C F_{n}, \varepsilon_{n}^{\prime}$ be a balayaged measure of the Dirac measure $\varepsilon$ at $P$ onto $F_{n}$ and $\gamma_{\varepsilon n}$ be an associated non-negative constant. $\varepsilon_{n}^{\prime}$ and $\mu_{n}^{\prime}$ being of finite logarithmic energy, we have

$$
\begin{aligned}
& U^{\mu_{n}^{\prime}}(P)-\gamma_{\mu_{n}}=\int U^{\varepsilon} d \mu_{n}^{\prime}-\gamma_{\mu n} \\
= & \int\left(U^{\varepsilon_{n}^{\prime}}-\gamma_{i n}\right) d \mu_{n}^{\prime}-\gamma_{\mu n} \\
= & \int\left(U^{\mu_{n}^{\prime}}-\gamma_{\mu n}\right) d \varepsilon_{n}^{\prime}-\gamma_{\varepsilon n} \\
= & \int\left(U^{\mu_{n+1}^{\prime}}-\gamma_{\mu(n+1)}\right) d \varepsilon_{n}^{\prime}-\gamma_{\varepsilon n} \\
= & \int\left(U^{\varepsilon_{n}^{\prime}}-\gamma_{\varepsilon n}\right) d \mu_{n+1}^{\prime}-\gamma_{\mu(n+1)} \\
= & \int U^{\varepsilon} d \mu_{n+1}^{\prime}-\gamma_{\mu(n+1)}=U^{\mu_{n+1}^{\prime}(P)-\gamma_{\mu(n+1)} .}
\end{aligned}
$$

The required inequality holds on $F_{n}$ with a possible exception of a set of logarithmic capacity zero. It holds everywhere on account of the superharmonicity of logarithmic potentials. We may suppose that $\left\{\mu_{n}^{\prime}\right\}$ is a vaguely convergent sequence by selecting its partial sequence in advance if necessary. The limiting measure $\mu^{\prime}$ is a positive measure supported by $F$ with total mass 1 , and we have

$$
U^{\mu^{\prime}}(P) \leqq \lim _{n \rightarrow+\infty} U^{\mu_{n}^{\prime}}(P)
$$

everywhere, the equality holding with a possible exception of a set of logarithmic capacity zero. So, the sequence $\left\{\gamma_{u n}\right\}$ is convergent. Its limit $\gamma_{\mu}$ is a nonnegative constant. The logarithmic capacity of $G_{n}=F-F_{n}$ decreasing to zero, 
we have

(1) $U^{\mu^{\prime}}(P)=U^{\mu}(P)+\gamma_{\mu}$ on $F$ with a possible exception of a set of logarithmic capacity zero, and

(2) $U^{\mu^{\prime}}(P) \leqq U^{\mu}(P)+r_{\mu}$ everywhere.

Let us remark that this balayaged measure $\mu^{\prime}$ is the vague limit of a sequence of positive measures $\mu^{\prime}$ with total mass 1 , which are supported by $F$ and of finite logarithmic energy, and which satisfy

$$
U^{\mu_{n}^{\prime}}(P)-\gamma_{\mu n} \uparrow U^{\mu^{\prime}}(P)-\gamma_{\mu}
$$

everywhere with a convergent sequence $\left\{\gamma_{\mu n}\right\}$ of non-negative numbers and its limit $\gamma_{\mu}$.

[IV] The case where $F$ is compact and $\mu$ is any positive measure.

Let $\mu_{1}$ and $\mu_{2}$ be the restricted measures of $\mu$ to $F$ and to $C F$ respectively, $a_{1}$ and $a_{2}$ be their total masses respectively and $\mu_{1}^{\prime}$ and $\mu_{2}^{\prime}$ be balayaged meas. ures, with total masses $a_{1}$ and $a_{2}$, of $\mu_{1}$ and $\mu_{2}$ onto $F$ respectively. The measure $\mu^{\prime}=\mu_{1}^{\prime}+\mu_{2}^{\prime}$ is evidently a balayaged measure of $\mu$ onto $F$.

[V] The reciprocal relation in case $F$ is compact.

We are going to prove that the reciprocal relation holds for balayaged measures obtained above. Let $\mu$ be any positive measure with total mass 1 and $\nu$ be any positive measure of finite logarithmic energy with total mass 1 . As stated above, there are three cases for a balayaged measure $\mu^{\prime}$ of $\mu$ onto $F$ :

(1) It is a positive measure with total mass 1 supported by $F$ and of finite logarithmic energy,

(2) It is the sum of positive measures $\mu_{n}^{\prime}$ supported by $F$ and of finite logarithmic energy,

(3) It is the vague limit of a sequence of positive measures $\mu_{n}^{\prime}$ with total mass 1 which are supported by $F$ and of finite logarithmic energy and which satisfy

$$
U^{\mu_{n}^{\prime}}(P)-\gamma_{\mu, n} \uparrow U^{\mu^{\prime}}(P)-\gamma_{\mu}
$$

everywhere with a convergent sequence $\left\{\gamma_{\mu n}\right\}$ of non-negative numbers and its limit $\gamma_{\mu}$.

Since $\nu^{\prime}$ is of finite logarithmic energy, we have 


$$
\int\left(U^{\nu^{\prime}}-\gamma_{\nu}\right) d \mu=\int U^{\mu} d \nu^{\prime}-\gamma_{\nu}=\int\left(U^{\mu^{\prime}}-\gamma_{\mu}\right) d \nu^{\prime}-\gamma_{\nu}=\int U^{\mu^{\prime}} d \nu^{\prime}-\gamma_{\mu}-\gamma_{\nu}
$$

On the other hand, it is easy to prove that

$$
\int\left(U^{\mu^{\prime}}-\gamma_{\mu}\right) d \nu=\int U^{\mu^{\prime}} d \nu^{\prime}-\gamma_{\mu}-\gamma_{\nu}
$$

For example, in cases (3) we have

$$
\begin{aligned}
& \int\left(U^{\mu^{\prime}}-\gamma_{\mu}\right) d \nu=\lim _{n \rightarrow+\infty} \int\left(U^{\mu_{n}^{\prime}}-\gamma_{\mu n}\right) d \nu \\
= & \lim _{n \rightarrow+\infty} \int U^{\nu} d \mu_{n}^{\prime}-\gamma_{\mu}=\lim _{n \rightarrow+\infty} \int\left(U^{\nu^{\prime}}-\gamma_{\nu}\right) d \mu_{n}^{\prime}-\gamma_{\mu} \\
= & \lim _{n \rightarrow+\infty} \int U^{\mu_{n}^{\prime}} d \nu^{\prime}-\gamma_{\mu}-\gamma_{\mu} \\
= & \lim _{n \rightarrow+\infty} \int\left(U^{\mu_{n}^{\prime}}-\gamma_{\mu n}+\gamma_{\mu n}\right) d \nu^{\prime}-\gamma_{\mu}-\gamma_{\nu} \\
= & \int\left(U^{\mu^{\prime}}-\gamma_{\mu}\right) d \nu^{\prime}-\gamma_{\nu}=\int U^{\mu^{\prime}} d \nu^{\prime}-\gamma_{\mu}-\gamma_{\nu} .
\end{aligned}
$$

It is proved similarly in cases (1) and (2).

[VI] The case where $F$ is a non-compact closed set and $\mu$ is any positive measure.

Let $S_{n}$ be a closed disk of radius $n$ centered at the origin, $\mu_{n}^{\prime}$ be a balayaged measure of $\mu$ onto $F_{n}=F \cdot S_{n}$ and $\gamma_{\mu n}$ be the associated non-negative constant. First, let us prove

$$
U^{\mu_{1}^{\prime}}(P)-\gamma_{\mu_{1}} \leqq U^{\mu_{2}^{\prime}}(P)-\gamma_{\mu_{2}} \leqq U^{\mu_{3}^{\prime}}(P)-\gamma_{\mu_{3}} \leqq \cdots \rightarrow U^{\mu}(P)
$$

everywhere. Let $P$ be any point of $C F_{n}, \lambda$ be the circular measure with total mass 1 on a small circle, outside $F_{n}$, with the center at $P, \lambda_{n}^{\prime}$ be a balayaged measure of $\lambda$ onto $F_{n}$ and $\gamma_{\lambda n}$ be an associated constant. Since both $\lambda$ and $\lambda_{n}^{\prime}$ are of finite logarithmic energy, we have

$$
\begin{aligned}
& U^{\mu_{n}^{\prime}}(P)-\gamma_{\mu n}=\int\left(U^{\mu_{n}^{\prime}}-\gamma_{\mu n}\right) d \lambda=\int\left(U_{n}^{\lambda_{n}^{\prime}}-\gamma_{\lambda n}\right) d \mu \\
= & \int U^{\mu} d \lambda_{n}^{\prime}-\gamma_{\lambda n}=\int\left(U^{\mu_{n}^{\prime}}-\gamma_{\mu n}\right) d \lambda_{n}^{\prime}-\gamma_{\lambda n} \\
= & \int\left(U^{\mu_{n+1}^{\prime}}-\gamma_{\mu(n+1)}\right) d \lambda_{n}^{\prime}-\gamma_{\lambda n} \\
= & \int U^{\lambda_{n}^{\prime}} d \mu_{n+1}^{\prime}-\gamma_{\lambda n}-\gamma_{\mu(n+1)}
\end{aligned}
$$




$$
\begin{aligned}
& \leqq \int\left(U^{\lambda}+\gamma_{\lambda n}\right) d \mu_{n+1}^{\prime}-\gamma_{\lambda n}-\gamma_{\mu(n+1)} \\
& \left.=\int\left(U^{\mu_{n+1}^{\prime}}-\gamma_{\mu(n+1)}\right) d \lambda=U^{\mu_{n+1}^{\prime}}(P)-\gamma_{\mu(n+1}\right) .
\end{aligned}
$$

The required inequality holds on $F_{n}$ with a possible exception of a set of logarithmic capacity zero. It holds everywhere on account of the superharmonicity of logarithmic potentials. By the integration with respect to the circular measure $\lambda$ with total mass 1 on a large circle of radius $R$ and with center at the origin, we have

$$
\log \frac{1}{R}-\gamma_{\mu n} \leqq \log \frac{1}{R}-\gamma_{\mu(n+1)}
$$

So, the sequence $\left\{\gamma_{\mu n}\right\}$ decreases to a non-negative number $\delta_{\mu}$ with $1 / n$ and $\lim U^{\mu_{n}^{\prime}}(P)>-\infty$ exists everywhere. Next, we choose a vaguely convergent subsequence of $\left\{\mu_{n}^{\prime}\right\}$. It will be denoted again by $\left\{\mu_{n}^{\prime}\right\}$. As $\left\{U^{\mu_{n}^{\prime}}(P)-\gamma_{\mu_{n}}\right\}$ is a sequence of superharmonic functions monotone increasing with $n$ and the limiting function is not identically equal to $+\infty$, it converges to a superharmonic function. Consequently $\lim U_{n}^{\mu_{n}^{\prime}}(P)$ is superharmonic. Take an increasing sequence $\left\{R_{k}\right\}$ of numbers such that each closed disk $S_{k}$ of radius $R_{k}$ centered at the origin has no positive mass for $\mu^{\prime}$ on its boundary. We have

$$
\lim _{n \rightarrow+\infty} \int_{S_{k}} \log \frac{1}{P Q} d \mu_{n}^{\prime}(Q)=\int_{S_{k}} \log \frac{1}{P Q} d \mu^{\prime}(Q)
$$

in the plane with a possible exception of a set of logarithmic capacity zero for each $k$. Let $M$ be a point inside $S_{1}$ at which the limit exists for all $k$. Since

$$
\lim _{n \rightarrow+\infty} U^{\mu_{n}^{\prime}}(M)
$$

exists,

$$
\lim _{n \rightarrow+\infty} \int_{c s_{k}} \log \frac{1}{M Q} d \mu_{n}^{\prime}(Q)
$$

exists for each $k$. This increases to a non-positive finite value as $k \rightarrow+\infty$. We shall denote it by $\alpha$. Take any compact set $K$ which contains a point $M$. We have

$$
\left|\int_{C S_{k}} \log \frac{1}{P Q} d \mu_{n}^{\prime}(Q)-\int_{C s_{k}} \log \frac{1}{M Q} d \mu_{n}^{\prime}(Q)\right| \leqq \int_{C s_{k}}\left|\log \frac{M Q}{P Q}\right| d \mu_{n}^{\prime}(Q)
$$


for any point $P$ of $K$ if $K \subset S_{k}$. If $R_{k}$ is large, $|\log M Q / P Q|$ is arbitrarily small for all $Q$ in $C S_{k}$. Hence, given $\varepsilon>0$, there are $n_{0}$ and $k_{0}$ such that

$$
\left|\int_{C S_{k}} \log \frac{1}{P Q} d \mu_{n}^{\prime}(Q)-\alpha\right|<\varepsilon \text { for } k \geqq k_{0} \text { and } n \geqq n_{0} .
$$

As we have

$$
\lim _{n \rightarrow+\infty} \int_{s_{k}} \log \frac{1}{P Q} d \mu_{n}^{\prime}(Q)=\int_{s_{k}} \log \frac{1}{P Q} d \mu^{\prime}(Q)
$$

in the plane with a possible exception of a set of logarithmic capacity zero, we have

$$
\left|\lim _{n \rightarrow+\infty}\left(U^{\mu_{n}^{\prime}}(P)-\int_{s_{k}} \log \frac{1}{P Q} d \mu^{\prime}(Q)-\alpha\right)\right|=\left|\lim _{n \rightarrow+\infty} \int_{C S_{k}} \log \frac{1}{P Q} d \mu_{n}^{\prime}(Q)-\alpha\right|<\varepsilon
$$

if $k$ is sufficiently large, where $\varepsilon>0$ is given. This shows that $U^{u^{\prime}}(P)=$ $\int \log 1 / P Q d \mu^{\prime}(Q)$ exists and equals $\lim U^{\mu_{n}^{\prime}}(P)-\alpha$ on $K$ and hence in the whole plane with a possible exception of a set of logarithmic capacity zero. Since $\lim U^{\mu_{n}^{\prime}}(P)$ is superharmonic in the plane, the equality holds without exception. We recall that $U^{\mu_{n}^{\prime}}(P)-\gamma_{\mu n} \leqq U^{\mu}(P)$ in the plane with the equality holding on $F$ possibly except for a set of logarithmic capacity zero. Now we have

(1) $U^{\mu^{\prime}}(P)-\gamma_{\mu}=U^{\mu}(P)$ on $F$ with a possible exception of a set of logarithmic capacity zero, where $\gamma_{\mu}=\delta_{\mu}-\alpha \geqq 0$, and

(2) $U^{\mu^{\prime}}(P)-\gamma_{\mu} \leqq U^{\mu}(P)$ everywhere.

We remark that the total mass of $\mu^{\prime}$ is one. To prove it we use the fact that

$$
\alpha=\lim _{k \rightarrow+\infty} \lim _{n \rightarrow+\infty} \int_{C s_{k}} \log \frac{1}{M Q} d \mu_{n}^{\prime}(Q)
$$

is a finite value. Since $M Q \geqq R_{k} / 2$ on $C S_{k}$ if $k$ is large,

$$
\alpha \leqq \lim _{k \rightarrow+\infty} \lim _{n \rightarrow+\infty} \log \left(2 / R_{k}\right) \mu_{n}^{\prime}\left(C S_{k}\right) .
$$

This shows that $\lim _{k \rightarrow+\infty} \lim _{n \rightarrow+\infty} \mu_{n}^{\prime}\left(C S_{k}\right)=0$, whence the total mass of $\mu^{\prime}$ is one.

[VII] The reciprocal relation in case $F$ is a non-compact closed set and the uniqueness of balayaged measures.

We are going to prove that the reciprocal relation holds for balayaged measures obtained above. Let $\mu$ be any positive measure with total mass 1 and $\nu$ be a positive measure of finite logarithmic energy with total mass 1 . Let $\left\{\mu_{n}^{\prime}\right\}$ and $\left\{\nu_{n}^{\prime}\right\}$ be the sequences of balayaged measures of $\mu$ and $\nu$ onto $F_{n}$ 
respectively and $\left\{\gamma_{\mu n}\right\}$ and $\left\{\gamma_{\nu n}\right\}$ be the sequences of their associated non-negative constants. We have as stated in [V]

$$
\int\left(U^{\mu_{n}^{\prime}}-\gamma_{\mu n}\right) d \nu=\int\left(U^{\nu_{n}^{\prime}}-\gamma_{\nu n}\right) d \mu
$$

As we have

$$
U^{\mu_{n}^{\prime}}(P)-\gamma_{\mu n} \uparrow U^{\mu_{n}^{\prime}}(P)-\gamma_{\mu}
$$

and

$$
U^{\nu_{n}^{\prime}}(P)-\gamma_{\nu n} \uparrow U^{\nu_{n}^{\prime}}(P)-\gamma_{\nu}
$$

everywhere, we have

$$
\int\left(U^{\mu^{\prime}}-\gamma_{\mu}\right) d \nu=\int\left(U^{\nu^{\prime}}-\gamma_{\nu}\right) d \mu
$$

Finally, let us consider the uniqueness of balayaged measures. Let $\mu^{\prime}$ and $\mu^{\prime \prime}$ be balayaged measures of $\mu$ onto $F$. Suppose that

(1) $U^{\mu^{\prime}}(P)=U^{\mu}(P)+\gamma_{\mu}^{\prime}$ and $U^{\mu^{\prime \prime}}(P)=U^{\mu}(P)+\gamma_{\mu}^{\prime \prime}$ on $F$ with a possible exception of a set of logarithmic capacity zero, and

(2) $U^{\mu^{\prime}}(P) \leqq U^{\mu}(P)+\gamma_{\mu}^{\prime}$ and $U^{\mu^{\prime \prime}}(P) \leqq U^{\mu}(P)+\gamma_{\mu}^{\prime \prime}$ everywhere, $\gamma_{\mu}^{\prime}$ and $\gamma_{\mu}^{\prime \prime}$ being non-negative constants.

For the circular measure $\lambda$ with total mass 1 on any closed circle centered at any point $P$, we have

$$
\int\left(U^{\lambda^{\prime}}-\gamma_{\lambda}\right) d \mu=\int\left(U^{\mu^{\prime}}-\gamma_{\mu}^{\prime}\right) d \lambda=\int\left(U^{\mu^{\prime \prime}}-\gamma_{\mu}^{\prime \prime}\right) d \lambda .
$$

So, we have

$$
\int\left(U^{\mu^{\prime}}-U^{\mu^{\prime \prime}}\right) d \lambda=\gamma_{\mu}^{\prime}-\gamma_{\mu}^{\prime \prime}
$$

which induces

$$
\int\left(U^{\mu^{\prime}}-U^{\mu^{\prime \prime}}\right)\left(d \lambda_{1}-d \lambda_{2}\right)=0
$$

for the circular measures $\lambda_{1}$ and $\lambda_{2}$ with total mass 1 on two concentric circles centered at $P$. Hence, we have

$$
\int U^{\lambda_{1}-\lambda_{2}} d \mu^{\prime}=\int U^{\lambda_{1}-\lambda_{2}} d \mu^{\prime \prime}
$$

which induces $\mu^{\prime}(S)=\mu^{\prime \prime}(S)$ for any disk $S$. In conclusion, we have $\mu^{\prime}=\mu^{\prime \prime}$ and $\gamma_{\mu}^{\prime}=\gamma_{\mu}^{\prime \prime}$. 
Definition. Let $F$ be any closed set. A point $P$ is called a regular point of $F$ if the balayaged measure $\varepsilon^{\prime}$ of the Dirac measure $\varepsilon$ at $P$ onto $F$ coincides with $\varepsilon$ and the associated non-negative constant $\gamma_{\varepsilon}$ reduces to zero.

With this terminology we have the following theorem.

Theorem 2. Two following expressions are equivalent.

[A] $A$ point $P$ is a regular point of $F$.

[B] Let $\mu$ be any positive measure with total mass 1, $\mu^{\prime}$ be the balayaged measure of $\mu$ onto $F$ and $\gamma_{\mu}$ be the associated non-negative constant. Then, it holds that

$$
U^{\mu^{\prime}}(P)=U^{\mu}(P)+\gamma_{\mu} .
$$

Proof. First, we prove that [A] implies [B]. Let $\lambda_{n}$ be the circular measure with total mass 1 on the closed circle of radius $1 / n$ centered at $P, \lambda_{n}^{\prime}$ be the balayaged measure of $\lambda_{n}$ onto $F$ and $\gamma_{\lambda n}$ be the associated non-negative constant. Let us remark that $U^{\lambda_{n}^{\prime}}-r_{\lambda n}$ increases to $U^{\varepsilon}$ with $n$ everywhere. It is because we have

$$
\int\left(U^{\lambda_{n}^{\prime}}-\gamma_{\lambda n}\right) d \lambda=\int\left(U^{\lambda^{\prime}}-\gamma_{\lambda}\right) d \lambda_{n}=\int U^{\lambda_{n}} d \lambda^{\prime}-\gamma_{\lambda}
$$

for the circular measure $\lambda$ with total mass 1 on any closed circle, the balayaged measure $\lambda^{\prime}$ of $\lambda$ onto $F$ and the associated non-negative constant $\gamma_{\lambda}$, and the quantity increases with $n$ to

$$
\int U^{\varepsilon} d \lambda^{\prime}-\gamma_{\lambda}=\int\left(U^{\lambda^{\prime}}-\gamma_{\lambda}\right) d \varepsilon=\int\left(U^{\varepsilon^{\prime}}-\gamma_{\varepsilon}\right) d \lambda=\int U^{\varepsilon} d \lambda .
$$

It follows that

$$
U^{\mu^{\prime}}(P)-\gamma_{\mu}=\lim _{n \rightarrow+\infty} \int\left(U^{\mu^{\prime}}-\gamma_{\mu}\right) d \lambda_{n}=\lim _{n \rightarrow+\infty} \int\left(U^{\lambda_{n}^{\prime}}-\gamma_{\lambda n}\right) d \mu=\int U^{\varepsilon} d \mu=U^{\mu}(P) .
$$

Next, we prove that [B] implies [A]. Let $\varepsilon^{\prime}$ be the balayaged measure of the Dirac measure $\varepsilon$ at $P$ onto $F$ and $\gamma_{\varepsilon}$ be the associated non-negative constant. We have

$$
\begin{aligned}
\int U^{\mu} d \varepsilon & =\int\left(U^{\mu^{\prime}}-\gamma_{\mu}\right) d \varepsilon=\int\left(U^{\varepsilon^{\prime}}-\gamma_{\varepsilon}\right) d \mu \\
& =\int U^{\mu} d \varepsilon^{\prime}-\gamma_{\varepsilon}
\end{aligned}
$$

for any positive measure $\dot{\mu}$ of finite logarithmic energy with total mass 1 . 
Therefore, we have

$$
\int U^{\lambda_{1}-\lambda_{2}} d \varepsilon=\int U^{\lambda_{1}-\lambda_{2}} d \varepsilon^{\prime}
$$

for any circular measure $\lambda_{1}$ and $\lambda_{2}$ with total mass 1 on two concentric closed circles, which implies $\varepsilon(S)=\varepsilon^{\prime}(S)$ for any disk $S$. So, we have $\varepsilon=\varepsilon^{\prime}$ and $\gamma_{\varepsilon}=0$.

Question. In Theorem 1, the associated non-negative constant $\gamma_{\mu}$ in the balayage of any positive measure $\mu$ onto any closed set $F$ does not always reduce to zero. But, if the complement of $F$ is bounded, the constant $\gamma_{\mu}$ reduces to zero. What conditions are necessary and sufficient for a closed set $F$ in order that the associated non-negative constant $\gamma_{\mu}$ in the balayage of any positive measure $\mu$ onto $F$ always reduces to zero?

\section{REFERENCES}

[1] O. Frostman.: Potentiel d'équilibre et capacité des ensembles avec quelques applications à la théorie des fonctions, Meddelanden Lunds Univ. Mat. Sem., Band 3, 1935.

[2] C. de la Vallée Poussin: Le potentiel logarithmique, Gauthier-Villars, Paris, 1949.

Department of Mathematics

Osaka City University 